\title{
Pyruvate May be a Novel Intervention of Diabetes
}

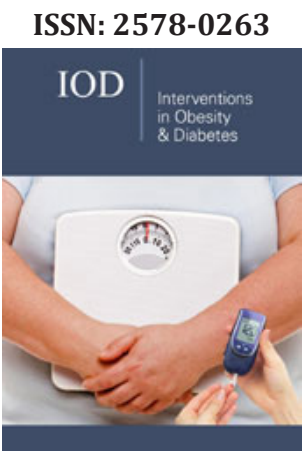

*Corresponding author: Fang-Qiang Zhou, 200 W. Sahara Ave., Unit 604, Las Vegas, NV 89102. Email: fqzh60130@ yahoo.com, phone: 1-708-785-3568.

Submission: 眥 February 18, 2021

Published: 眥 March 04, 2021

Volume 5 - Issue 1

How to cite this article: Fang-Qiang Zhou. Pyruvate May be a Novel Intervention of Diabetes. Interventions Obes Diabetes 5(1). IOD. 000602. 2021.

DOI: 10.31031/IOD.2021.05.000602

Copyright@ Fang-Qiang Zhou. This article is distributed under the terms of the Creative Commons Attribution 4.0 International License, which permits unrestricted use and redistribution provided that the original author and source are credited.

\author{
Fang-Qiang Zhou* \\ Fresenius Medical Care, Dialysis Centers in Chicago, USA
}

\begin{abstract}
Recent advances in pyruvate studies indicate that pyruvate may be a novel therapy in care of diabetes and its organ complications. The major action of pyruvate protection against diabetes may be in rejuvenation of glucose oxidation by preserving glycolysis and reactivating pyruvate dehydrogenase activity in the tricarboxylic acid cycle, reversing the Warburg effect in diabetic glucometabolic disorders. Pyruvate preservation of glucose metabolism plus its beneficial effects, such as anti-hypoxia, -oxidative stress, -acidosis, -apoptosis and -advanced glycation end products and stimulation of insulin secretion, may turn diabetic vicious circle virtuous in initiation and development of diabetic process. This review proposed a novel opinion focused on pyruvate superior biomedical and pharmacological properties in diabetes treatment and experimental and clinical evidence of pyruvate intervention in diabetes. The novel pyruvate modified oral rehydration salt (Pyr-ORS), based on WHO-ORS, may be helpful in the prevention and treatment of diabetes in a large population. Further studies and clinical trials are urgently required.
\end{abstract}

Keywords: Acidosis; Diabetes; Hypoxia; Oral rehydration salt; Pyruvate

Abbreviations: AGEs: Advanced Glycation End Products; LDH: Lactate Dehydrogenase; PDH: Pyruvate Dehydrogenase; PK: Pyruvate Kinase; TCA: Tricarboxylic Acid; TDD: Total Daily Dose

\section{Introduction}

Pyruvate of sodium salt has been studied, in vitro and in vivo, in animals and humans since as early as the 1930s. In past over half a century it was extensively and intensively investigated in laboratories as well as clinical tests. Numerous findings demonstrate that pyruvate is a unique anion, superior to prevailing anions in current medical fluids: acetate, bicarbonate, chloride, citrate, gluconate, lactate, phosphate and even malate [1]. The paramount biomedical advantages of supraphysiological doses of exogenous pyruvate are potently protective of organ metabolism and function in various pathogenic attacks, including anoxia, hypoxia/ischemia, trauma/burn, infection/sepsis, hypo/hyperglycemia or diabetes and even poisoning. The beneficial biological and pharmacological features underlying the cellular and molecular mechanisms of pyruvate protection from a variety of pathogenic assaults, specifically as to high glucose or diabetes [2,3], are summarized below.

\section{Pyruvate Beneficial Metabolic Attributes in Diabetes}

\section{Enhancing anoxia/hypoxia tolerance}

By the reductive reaction with Lactate Dehydrogenase (LDH) free of energy, exogenous pyruvate reduction spontaneously coupled with Nicotinamide Adenine Dinucleotide reduced form (NADH) oxidation generates lactate and oxidized form ( $\left.\mathrm{NAD}^{+}\right)$on an equal molecular basis in anoxic conditions, consuming hydrogen (proton, $\left[\mathrm{H}^{+}\right]$) in cytosol. Thus, the $\mathrm{LDH}$ reduction is a systemic alkalizing reaction throughout the body; the reaction results in raising the $\mathrm{NAD}^{+}$/ NADH ratio that is essential for glycolysis at Glyceraldehyde 3-Phosphate Dehydrogenase (G-3PD) and improving glycolysis-rate limiting enzymes, like Phosphofructokinase-1 (PFK-1) and Pyruvate Kinase (PK), which are $\mathrm{pH}$-sensitive, contributing to preservation of canonical glycolytic pathways $[1,4,5]$. Therefore, exogenous pyruvate maintains anaerobic glycolysis and glycolytic-ATP generation that are indispensable for cellular basic activities, such as $\mathrm{Na}^{+}-\mathrm{K}^{+}-\mathrm{ATPase}$ and islet insulin secretion [6-8]. Further, exogenous pyruvate can reactivate the depressed Pyruvate Dehydrogenase (PDH) activity-induced by various insults, including hypoxia and high glucose or diabetes, via the direct inhibition of promoted PDH Kinase (PDK) activity as a PDH stimulator like Dichloroacetate (DCA) free of oxygen $[3,4,9]$. Furthermore, 
pyruvate also prompts the anaplerotic flux via the Pyruvate Carboxylase (PC) in the Tricarboxylic Acid (TCA) cycle though no direct evidence yet in diabetes $[4,10]$. As a result, pyruvate additionally as an energy substrate stimulates the TCA cycle in mitochondria, enhancing lactate oxidation and mitochondrial ATP production in hypoxic/ischemic and diabetic conditions. Notably, diabetes may initiate with the PK and PDH inhibition, leading to glucometabolic disorders as the Warburg effect as pseudohypoxia with the NADH/NAD ${ }^{+}$rise [11,12]. Hypoxia in tissues is also involved in diabetes development. Accordingly, exogenous pyruvate can improve these aberrant metabolic alterations, promoting glucose oxidation and reversing the Warburg effect $[3,11,12]$. A scheme of the post-pyruvate metabolic profile was illustrated previously [1,35].

\section{Correcting severe acidemia}

Hypoxic lactic acidosis and diabetic ketoacidosis, which both are fatal complications of critical illnesses with or without diabetes. By the renovation of lactate and ketone oxidation in the TCA cycle with pyruvate stimulated $\left.\mathrm{PDH}, \mathrm{H}^{+}\right]$is consumed in addition to the $\mathrm{LDH}$ reduction with $\left[\mathrm{H}^{+}\right]$consumption. Besides, pyruvatebased gluconeogenesis in cytosol is additionally a process of $\left[\mathrm{H}^{+}\right]$ consumption, thereby preventing $\left[\mathrm{H}^{+}\right]$accumulation in hypoxia/ ischemia [1,4]. Alternatively, the lower pyruvate dissociation constant (pKa) of 2.49 (weaker buffering capacity) quickly facilitates a higher intracellular $\mathrm{pH}$ [4]. Therefore, only can pyruvate correct hypoxic lactic acidosis with survival improvement in resuscitation from severe shock, neither lactate, nor citrate, acetate or bicarbonate can do in shocked animals, despite equimolar alkalizers administration [13-15]. It is worthy to note that diabetic patients with ketoacidosis show more lower PDH activity relative to non-ketoacidosis patients [3]. It is potentially perspective that intravenous pyruvate may cure ketoacidosis and lactic acidosis in diabetic patients even without insulin addition in selective clinical conditions $[3,4]$.

\section{Exerting powerful anti-oxidative stress/inflammation/ apoptosis}

Exogenous pyruvate can directly react with reactive oxygen and nitrogen species (oxidative or nitrosative stress) in a nonenzymatic and stoichiometric manner, producing carbon dioxide or acetate and water. It also indirectly exerts as an anti-oxidation by increasing the redox potentials. In addition, pyruvate is an inhibitor of inflammatory mediators (cytokines, TNF- $\alpha$ and NF-kB) and inflammatory cell (neutrophils) infiltration in tissues $[3,16]$. Also, pyruvate protects mitochondrial function and endoplasmic reticulum (ER) from stress and apoptosis induced by hypoxia and high glucose [2,9]. Importantly, all these pathogenic factors participate in the initiation and progression of diabetes, specifically Type $2[3,11]$.

\section{Stimulating insulin secretion from islets and inhibiting AGEs}

Other than previous conception, recent findings are preliminarily considered effective of sodium pyruvate as an insulin- stimulator. Pyruvate metabolism in mitochondria is critically involved in glucose-stimulated insulin secretion (GSIS) and the PC that pyruvate partially depend son in enhancement of the anaplerotic flux also plays a pivotal role in insulin secretion from islets $[17,18]$. Thus, exogenous pyruvate facilitates insulin secretion in islet $\beta$-cells even in Type 1 diabetic patients $[19,20]$. On the other hand, several findings demonstrate that pyruvate attenuates advanced glycation end products (AGEs) generation and tissue deposition, which is one of major pathogenic triggers in organ complications, in hypoxic or diabetic tissues [3,21,22]. Evidently, both unique characteristics of pyruvate are of robust clinical significance in diabetic reversal. All above pathogenic factors and $\beta$-cells disorders as well as insulin resistance are intricately interacted as a vicious cycle in initiating and developing diabetes and its organ complications. In contrast, exogenous pyruvate multifactorial protection against the above alterations, specially from the glucometabolic disorders by restoration of PK, PDH and PC activities to sustain the normal glucose oxidation in the TCA cycle and mitochondrial function, reverses the Warburg effect. Therefore, pyruvate as DCA and its new derivatives may be a novel intervention of obesity and diabetes and its organ complications by rejuvenation of glucose oxidation, turning diabetic vicious cycle virtuous, other than the current concept with restriction of carbohydrate intake (weight loss and bariatric surgery) plus GLP-1 (glucagon-like peptide-1) receptor agonists and increase of glucosuria with SGLT-2 (sodium glucose linked transporter-2) inhibitors [3].

\section{Evidence and Possibility of Pyruvate Effects on Diabetes}

Many animal experiments and clinical tests have demonstrated that exogenous pyruvate is beneficial in caring clinical diabetes. In 1990s, it was first found that pyruvate prolonged human pancreatic islets viability and protected from diabetic cataract both in, in vitro, experiments $[23,24]$. Subsequent studies not only indicated that pyruvate is an effective therapy in islet transplantation in diabetic rats, but also substantiated that oral pyruvate attenuates diabetic cataract after onset of cataract in, in vivo, investigations [21,25]. These findings were duplicated and further studies displayed that oral pyruvate effectively protected from diabetic retinopathy in rats $[26,27]$. Although lack of studies on pyruvate effects on diabetic islets in vivo, a recent experiment showed the pyruvate treatment preserved insulin secretion from $\beta$-cells and avoided hyperglycemia in a non-diabetic rat model with multiple organ failure syndrome-induced by burn and septic injuries [28]. Newly, a human renal tubular epithelial cell-line (HK-2) was showed as a robust pyruvate protection in high glucose cultures, demonstrating the effectiveness against ER stress and apoptosis induced by high glucose [2]. Notably, as illustrated in diabetic oculopathy, oral pyruvate in drink water also revealed a significant therapeutic effect on diabetic nephropathy accompanied with the fast blood sugar/insulin decrease/increase during an 8-week treatment in $\boldsymbol{d} \boldsymbol{b} / \boldsymbol{d} \boldsymbol{b}$ mice [3]. It is worthy of noting that albeit just a few of clinical case reports, oral pyruvate with a large dose daily (30-60g/d for 7-10d) efficiently controlled 6 patients with Type 1 diabetes. The clinical signs of hypoglycemia with the decrease in postprandial 
blood sugar were observed and 4 of them had to reduce the Total Daily Dose (TDD) of insulin injection due to hypoglycemia [19]. One case with mitochondrial diabetes orally ingested pyruvate $(0.5 \mathrm{~g} /$ $\mathrm{kg}$, thrice daily for 10 months), improved clinical symptoms and reduced insulin TDD from $32 \mathrm{IU} / \mathrm{d}$ to $20 \mathrm{IU} / \mathrm{d}$ after the treatment for 6 months followed by $22 \mathrm{IU} / \mathrm{d} 2$ months after the termination of therapy [20]. Interestingly, 10 children with citrin-deficiency displayed a significant rise of fasting insulin secretion after oral pyruvate treatment $(0.3 \mathrm{~g} / \mathrm{kg} / \mathrm{d})$ for $3-6$ months without fasting blood sugar changed [29].

Despite the long history of pyruvate in food supplemental markets for weight loss and in nutrition textbooks for obesity with a question marker, it has been well known recently that oral single pyruvate dosage between $7-25 \mathrm{~g} / \mathrm{d}$ does not enhance blood/ plasm pyruvate levels and effects are limited due to malabsorption, but the large dosage shows gastrointestinal irritation in humans [1]. Fortunately, a novel low pyruvate in pyruvate-enriched oral rehydration salt/solution (Pyr-ORS, pyruvate in 3.5g/L with glucose in 13.5-20.0g/L) was innovated in 2012 [30,31], i.e., pyruvate in Pyr-ORS substitutes the equimolar alkalizers: bicarbonate or citrate in WHO-ORS that saves a couple of million lives of young patients suffered from acute diarrhea or cholera worldwide yearly since 1970s [1]. In comparison, Pyr-ORS profoundly prompts intestinal absorption of sodium and water along with pyruvate and protects systemic cardiovascular dynamics, visceral organ and intestinal barrier function with a marked rise of blood pyruvate, resulting in correcting lethal lactic acidosis and prolonging survival in enteral rehydration of shocked animals superior to WHO-ORS, which was developed according to the physiological basis of the $\mathrm{Na}^{+}$-glucose co-transporter in intestinal epithelium in 1950s [1,14,15,30-32]. Newly, findings of Pyr-ORS robust protection against diabetic nephropathy in diabetic $\boldsymbol{d} \boldsymbol{b} / \boldsymbol{d} \boldsymbol{b}$ mice and acute brain injury after cardiac arrest in rats strongly suggest its potential clinical value in fighting diabetes and aging, like Alzheimer's disease [3,33,34]. Besides intravenous pyruvate would benefit in critical care diabetic patients with or without lactic acidosis and ketoacidosis, pyruvate may be also advantageous over bicarbonate (at least partial replacement) in hemodialysis solutions and lactate in peritoneal dialysis solutions, specifically in diabetic patients [35,36]. In addition, Pyr-ORS may be useful in geriatric and sport medicine. In these respects, further intensive research is valuable. Its clinical safety and feasibility concerning, in vivo, cytotoxicity and longterm stability in aqueous solutions were recently discussed with a favorable prospect $[1,37]$.

\section{Conclusion}

Pyruvate has beneficial effects on clinical diabetes treatment. The key pyruvate action may rejuvenate glucose oxidation in diabetes, making diabetic reversal. Oral Pyr-ORS (the formula may be improved as needed, and/or by adding favorable additives) as a novel approach or a functional drink may be helpful in prevention and treatment of diabetes and its organ complications in a large population. Pyruvate applications would indicate a novel superior medical intervention in clinical settings, specifically in diabetes treatment. Further studies and clinical trials are urgently warranted in diabetes as well as in critical care patients.

\section{Authors Declaration}

The author declares that there is no conflict of interest. There is not funding provided for this paper.

\section{Note}

The opinions or assertions contained herein are not a reflection of the view of Fresenius Medical Care, Dialysis Centers in Chicago, Illinois.

\section{References}

1. Zhou FQ (2020) Pyruvate research and clinical application outlooks a revolutionary medical advance. Int J Nutr 5: 1-8.

2. Zhang XM, Wang YZ, Tong JD, Ning XC, Zhou FQ, et al. (2020) Pyruvate alleviates high glucose-induced endoplasmic reticulum stress and apoptosis in HK-2 cells. FEBS Open Bio 10(5): 827-834.

3. Zhang XM, Deng H, Tong JD, Wang YZ, Ning XC, et al. (2020) Pyruvateenriched oral rehydration solution improves glucometabolic disorders in kidneys of diabetic $d b / d b$ mice. J Diabetes Res.

4. Zhou FQ (2005) Pyruvate in the correction of intracellular acidosis: A metabolic basis as a novel superior buffer. Am J Nephrol 25(1): 55-63.

5. Wang Y, Huang Y, Yang J, Zhou FQ Zhao L, et al. (2018) Pyruvate is a prospective alkalizer to correct hypoxic lactic acidosis. Mil Med Res 5(1): 13.

6. Gou DM, Tan HJ, Cai HJ, Zhou FQ (2012) Pyruvate effects on red blood cells during in vitro cardiopulmonary bypass with dogs blood. Artif Organs 36(11): 988-991.

7. Van Emous JG, Vleggeert Lankamp CL, Nederhoff MG, Ruigrok TJ, Van Echteld CJ (2001) Postischemic $\mathrm{Na}^{+}-\mathrm{K}^{+}$-ATPase reactivation is delayed in the absence of glycolytic ATP in isolated rat hearts. Am J Physiol Heart Circ Physiol 280(5): H2189-H2195.

8. Pizarro Delgado J, Deeney JT, Corkey BE, Tamarit Rodriguez J (2016) Direct stimulation of islet insulin secretion by glycolytic and mitochondrial metabolites in KCl-depolarized islets. PLoS One 11(11): e0166111.

9. Sharma P, Walsh KT, Kerr Knott KA, Karaian JE, Mongan PD (2005) Pyruvate modulates hepatic mitochondrial functions and reduces apoptosis indicators during hemorrhagic shock in rats. Anesthesiology 103(1): 65-73.

10. Ledee DR, Kajimoto M, O Kelly Priddy CM, Olson AK, Isern N, et al. (2015) Pyruvate modifies metabolic flux and nutrient sensing during extracorporeal membrane oxygenation in an immature swine model. Am J Physiol Heart Circ Physiol 309(1): H137-H146.

11. Qi W, Li Q Gordin D, King GL (2018) Preservation of renal function in chronic diabetes by enhancing glomerular glucose metabolism. J Mol Med (Berl) 96(5): 373-381.

12.Xi L, Chow CM, Kong X (2016) Role of tissue and systemic hypoxia in obesity and type 2 diabetes. J Diabetes Res 2016: 1527852.

13. Hu S, Bai XD, Liu XQ, Wang HB, Zhong YX, et al. (2013) Pyruvate ringer's solution corrects lactic acidosis and prolongs survival during hemorrhagic shock in rats. J Emerg Med 45(6): 885-893.

14. Yu W, Hu S, Xie ZY, He ZJ, Luo HM, et al. (2015) Pyruvate oral rehydration solution improved visceral function and survival in shock rats. J Surg Res 193(1): 344-354.

15. Liu R, Wang SM, Li ZY, Yu W, Zhang HP, et al. (2018) Pyruvate in reduced osmolarity oral rehydration salt corrected lactic acidosis in sever scald rats. J Surg Res 226: 173-180. 
16. Flaherty DC, Hoxha B, Sun J, Gurji H, Simecka JW, et al. (2010) Pyruvatefortified fluid resuscitation improves hemodynamic stability while suppressing systemic inflammation and myocardial oxidative stress after hemorrhagic shock. Mil Med 175(3): 166-172.

17. Patterson JN, Cousteils K, Lou JW, Manning Fox JE, MacDonald PE, et al. (2014) Mitochondrial metabolism of pyruvate is essential for regulating glucose-stimulated insulin secretion. J Biol Chem 289(19):13335-13346.

18. Xu J, Han J, Long YS, Epstein PN, Liu YQ (2008) The role of pyruvate carboxylase in insulin secretion and proliferation in rat pancreatic beta cells. Diabetologia 51(11): 2022-2030.

19. Petkova I, Hristov V, Petrov K, Thorn W (2007) Oral application of sodium pyruvate in healthy persons and patients with diabetes mellitus type 1 Proceedings of the Bulgarian Academy of Sciences Clinical Medicine 60: 579-584.

20. Inoue T, Murakami N, Ayabe T, Oto Y, Nishino I, et al. (2016) Pyruvate improved insulin secretion status in a mitochondrial diabetes mellitus patient. J Clin Endocrinol Metab 101(5): 1924-1926.

21. Varma SD, Hegde KR, Kovtun S (2005) Attenuation and delay of diabetic cataracts by antioxidants: Effectiveness of pyruvate after onset of cataract. Ophthalmologica 219(5): 309-315.

22. Scott GF, Nguyen AQ Cherry BH, Hollrah RA, Salinas I, et al. (2017) Featured article: Pyruvate preserves antiglycation defenses in porcine brain after cardiac arrest. Exp Biol Med (Maywood) 242(10): 10951103.

23. Rastellini C, Cicalese L, Zeevi A, Mattes C, Stanko RT, et al. (1995) Longterm culture of viable human pancreatic islets in pyruvate-rich medium Transplant Proc 27(6): 3383-3384.

24. Beyer Mears A, Diecke FP, Mistry K, Ellison C, Cruz E (1997) Effect of pyruvate on lens myo-inositol transport and polyol formation in diabetic cataract. Pharmacology 55(2): 78-86.

25. Brown ML, Braun M, Cicalese L, Rastellini C (2005) Effect of perioperative antioxidant therapy on suboptimal islet transplantation in rats. Transplant Proc 37(1): 217-219.

26. Varma SD, Chandrasekaran K (2015) High sugar-induced repression of antioxidant and anti-apoptotic genes in lens: Reversal by pyruvate. Mol Cell Biochem 403(1-2): 149-158.
27. Qi YX, Fu JD, Wang YQ, Wang DL (2014) Effects of pyruvate on retinal oxidative damage and retinal ultrastructure in diabetic rats. Int Eye Sci 14: 2143-2146 (in Chinese with English abstract).

28. Ning LP, Wang ZK, Zhou FQ, Guo J, Wang Ql, et al. (2020) Protective effect of sodium pyruvate on islet $\beta$-cell dysfunction in the mice with multiple organ dysfunction syndrome induced by severe scald. Med \& Pharm J Chin PLA 32(7): 11-15 (in Chinese with English abstract).

29. Nagasaka H, Komatsu H, Inui A, Nakacho M, Morioka I, et al. (2017) Circulating tricarboxylic acid cycle metabolite levels in citrin-deficient children with metabolic adaptation, with and without sodium pyruvate treatment. Mol Genet Metab 120(3): 207-212.

30. Hu S, Liu WW, Zhao Y, Lin ZL, Luo HM, et al. (2014) Pyruvate-enriched oral rehydration solution improved intestinal absorption of water and sodium during enteral resuscitation in burns. Burns 40(4): 693-701.

31. Hu S, Lin ZL, Zhao ZK, Liu R, Ma L, et al. (2016) Pyruvate is superior to citrate in oral rehydration solution in the protection of intestine via hypoxia-inducible factor-1 activation in rats with burn injury. JPEN J Parenter Enteral Nutr 40(7): 924-933.

32. Liu R, Hu XH, Wang SM, Guo SJ, Li ZY, et al. (2016) Pyruvate in oral rehydration salt improves hemodynamics, vasopermeability and survival after burns in dogs. Burns 42(4): 797-806.

33. Bai WP, Li J, Han RL, Gu Y, Sun XD, et al. (2017) Effects of hypotonic pyruvate oral rehydration solution on brain injury in rat subjected to asphyxia cardiac arrest. Chin J Injury Repair and Wound Healing 12(5): 326-330 (in Chinese with English abstract).

34. Koivisto H, Leinonen H, Puurula M, Hafez HS, Barrera GA, et al. (2017) Corrigendum: Chronic pyruvate supplementation increases exploratory activity and brain energy reserves in young and middle-aged mice. Front Aging Neurosci 9: 67.

35. Wathen RL, Ward RA, Harding GB, Meyer LC (1982) Acid-base and metabolic responses to anion infusion in the anesthetized dog. Kidney Int 21(4): 592-599.

36. Zhou FQ (2001) Advantages of pyruvate over lactate in peritoneal dialysis solutions. Acta Pharmacol Sin 22(5): 385-392.

37. Zhou FQ (2020) Pyruvate potential effects on Covid-19 virus infection: Novel fluid intervention and prevention. London J Med Health Res 20(6): 69-77.

For possible submissions Click below: 